\title{
Bashingantahe - Traditionelle Gerichte in Burundi mit unterbrochener Tradition
}

\author{
Von Volker Lohse, Bielefeld
}

\section{Republik Burundi}

Die ostafrikanische Republik Burundi (Republika y'u Burundi / République du Burundi) (BU) gehört mit 27834 qkm$^{1}$ flächenmäßig zu den kleinsten Staaten Afrikas; mit rund 7 Mio Einwohnern (also etwa 245 je qkm) ist sie aber einer der am dichtesten besiedelten des Kontinents. ${ }^{2}$ Die Hauptstadt Bujumbura zählt über 300000 Einwohner, die Stadt Gitega ca. 110 000; die meisten Burunder leben allerdings in lockeren Streusiedlungen (rugos) zumeist auf den "1001 Hügeln" des Landes. ${ }^{3}$ Rund $82 \%$ der Einwohner sind Hutu (Bahutu), 14\% Tutsi (Watussi), 2\% Rundi und 2\% Twa-Pygmäen (Batwa); über 50\% der Bevölkerung ist jünger als 15 Jahre. $78 \%$ der Burunder sind katholische ${ }^{4}, 5 \%$ evangelische Christen; $13 \%$ hängen Naturreligionen an und 4\% sind Moslems. Die Burunder sprechen eine einheitliche Sprache, das Kirundi, eine Bantusprache, die die Tutsi von den Hutu übernahmen; zweite Amtssprache ist - wallonisch geprägtes - Französisch. Viele Einwohner sprechen zusätzlich Kisuaheli, wenige Twa.

Burundi hat Hochlandcharakter (bis zu 2670 m) mit einem Hochplateau auf $1700 \mathrm{~m}$ und besitzt nur einen kleinen Anteil am ostafrikanischen Graben. Die durchschnittliche Höhe von $1500 \mathrm{~m}$ üNN und das äquatoriale Regenklima erlauben die Nutzung von etwa $86 \%$ der Landfläche (25967 qkm) für Ackerbau oder als Weideland. Erzeugnisse für die Selbstversorgung nehmen etwa $94 \%$ der landwirtschaftlichen Nutzfläche in Anspruch. Als von Ruanda, Tansania und der Demokratischen Republik Kongo umgebenes Binnenland ${ }^{5}$ muss Burundi seine Importe bzw. Exporte (im Wesentlichen: Kaffee, Tee, Baumwolle) über den Land- oder den Luftweg abwickeln.

Das entspricht etwa der Größe des Landes Brandenburg.

2 Näher: Klingebiel, Stephan, Burundi, in: Nohlen, Dieter / Nuscheler, Franz: Handbuch der Dritten Welt Band 5, S. 54-67. Der Bevölkerungszuwachs beträgt etwa 2,2\% (1980-1990: 2,8\%).

3 Näher u.a. Bidou, J.-E./Ndayirukiye, S./Ndayishimiye, J.-P./Sirven, P., Géographie du Burundi, Paris 1991, passim m.w.N.

4 Die katholische Kirche erreichte in Burundi (und Ruanda) die größten Erfolge bei ihrer Mission in Afrika.

Die Entfernung zum Indischen Ozean beträgt in der Luftlinie 1100 km, zum Atlantik 1900 km. 
Burundi gehört zu den ärmsten Ländern der Erde und sein BIP/Einwohner/Jahr ist in den vergangenen, von Bürgerkriegswirren geprägten Jahren von 597 US\$ auf - geschätzt - 105 US\$ zurückgegangen.

Im 17. Jahrhundert gründeten die viehzüchtenden Tutsi-Einwanderer ihre Herrschaft über die alteingesessenen bäuerlichen Hutu, die bis zum frühen 14. Jahrhundert eingewandert waren und die ursprünglichen Bewohner, die pygmoiden Twa unterworfen oder verdrängt hatten. Die Unterwerfung der Hutu durch die Tutsi gelang relativ leicht, weil die Hutu politisch und militärisch zersplittert waren; das Dorf war ihre größte politische Einheit.

Historisch war das Land also bereits jahrhundertelang selbständiges Königreich, als es als Urundi nach 1884/85 Teil des deutschen Schutzgebiets Deutsch-Ostafrika wurde. ${ }^{6}$ Burundi hat also keine vom Kolonialherrn gezogenen Grenzen. Auch ließ die deutsche Kolonialverwaltung die vorgefundenen Herrschaftsstrukturen weitgehend unverändert (indirect rule): ${ }^{7}$

Von den an der Spitze der sozialen Hierarchie der damals ca. 1,5 Mio Einwohner stehenden ca. 200 Tutsifamilien stellte der Clan der Baganwa, die Königsfamilie, den Mwami (= umwami $=$ König $=$ der zur obersten Macht [Souveränität] Gekommene, Plural: Bami $)^{8}$, der auch nyen'ingoma (Herr der heiligen Trommel) oder umviyeyi (Vater, Herr) genannt wurde. Der Mwami war oberster Kriegs- und Gerichtsherr. Dem König stand ein Staatsrat aus den Clanältesten (abagabo) und besonders angesehenen und reichen Männern zur Seite. Das Königsamt hatte auch hohe sakrale Bedeutung; in diesem Bereich wurde der Mwami vom obersten Priester (umufumu w'umwami) und dem obersten Magier (Kiranga) beraten. Dem König gehörten die Leute, das Land und das Vieh, die er in dem komplizierten Feudalsystem als Lehen gab, dessen Heimfall nicht genau geregelt war. Zu Zeiten schwacher Könige lag die tatsächliche Macht bei den Chefs des Königsclans.

Nur die Baganwa und Angehörige von vier mächtigen, hochadeligen Ganwa-Familien durften wichtige Positionen in Verwaltung und Armee einnehmen. Das Königreich war in

Vgl. statt vieler: Bald, Detlef, Deutsch-Ostafrika 1900-1914, München 1970, passim m.w.N. und Hubatsch, Walther, Die Schutzgebiete des Deutschen Reiches 1884-1920, Band 22, Marburg/Lahn 1984, S. 364-423 (über die Residentur Urundi S. 419-421) m.w.N., von Spalding, R., Deutsch-Ostafrika, in: Schnee, Heinrich (Hrsg.), Deutsches Koloniallexikon, Band 1, Leipzig 1920, S. 357-407, ebda Uhlig, C., Urundi, Band 3, S. 587 f.

Näher: Meyer, Hans, Die Barundi. Eine völkerkundliche Studie aus Deutsch-Ostafrika, Leipzig 1916, passim (französische Fassung: Les Barundi, Paris 1984).

8 Regelmäßig folgte in der Erbmonarchie der älteste Sohn der Hauptfrau dem Vater auf dem Thron. Dabei gab es aber viele Ausnahmen, in denen der König einen anderen Sohn als Nachfolger bestimmte, näher: Mworoha, Émile, Histoire du Burundi des origines à la fin du XIXe siècle, Paris 1987, passim, besonders S. $105 \mathrm{ff}$. 
Provinzen eingeteilt, die von aus diesen Clans stammenden, belehnten Gouverneuren beherrscht wurden, die man nicht nur wegen der faktischen Erblichkeit dieser Machtposition auch als Provinzfürsten bezeichnen könnte. Ein Regional-Ältestenrat beriet sie. Provinzuntergliederungen wurden grundsätzlich von belehnten, einfachen Tutsibeamten (abatware oder batwale) verwaltet; ausnahmsweise hatten im Südosten Urundis Hutubeamte diese erbliche Funktion inne. Ein Kommunal-Ältestenrat beriet sie. Die Batwale übten auch die Justiz nach Gewohnheitsrecht aus.

Das Eigentum an Kühen war den Tutsi vorbehalten ${ }^{9}$, die Teile ihres Viehbestands an Hutu verpachteten. Diese Hutu gerieten dadurch in ein starkes Abhängigkeitsverhältnis zu den Tutsi. Ein kompliziertes Lehnssystem (Ubuhake bzw. Ubugabire) entstand auch auf dieser Ebene und sicherte dem Verpächter/Lehnsgeber/Lehnsherrn Einkünfte, Arbeitskräfte und Unterstützung bei Kriegszügen; der Pächter/Lehnsmann/Vasall erhielt Schutz, Unterstützung bei gerichtlichen Verfahren und Befreiung von der Blutrache. Hutu ohne Lehnsvertrag waren der Willkür der Verwaltungsbeamten des Mwami ausgesetzt, weil sie als vogelfrei galten. ${ }^{10}$

Im Justizbereich gab es Bashingantahe (Friedensgerichte) auf allen Ebenen; einzelne Mushingantahe (Friedensrichter) stiegen zu persönlichen Beratern des Königs auf (näher unten 3).

Als Belgien als Siegermacht des 1. Weltkriegs im Jahre 1920 das vom britischen Mandatsgebiet Tanganyika, dem größten Teil des früheren Deutsch-Ostafrika, abgetrennte Burundigebiet als Völkerbundsmandat übernahm, führte es neben dem lokalen Gewohnheitsrecht belgisches bzw. im Belgischen Kongo angepasstes Recht ("modernes Recht") ein, ${ }^{11}$ behielt aber das indirect-rule-System im Prinzip bei. ${ }^{12}$ Das belgische Völkerbundsmandat wurde 1923 formell bestätigt, und Belgien verwaltete das Mandatsgebiet Ruanda-Urundi gemeinsam mit dem Kongo. Im Jahre 1946 übernahmen die Vereinten Nationen das Treuhandsystem.

Im Gebiet am Tanganyikasee begannen 1952 erste Unabhängigkeitsbestrebungen, die am 1 . Juli 1962 - nach erheblichen Schwierigkeiten zwischen der Mandatsmacht und den Burundern vor allem von 1960 an - zur Unabhängigkeit der beiden jetzt getrennten Gebiete

9

10

11

12

Rindvieh hatte in Burundi neben dem wirtschaftlichen einen großen kultischen Wert.

Hausner, K.-H./Jezic, B., Rwanda, Burundi, Bonn 1968, S. 110; vgl. auch Seitz, St., Historische Wurzeln der ethnischen Spannungen in Burundi, in: Afrika Jahrbuch 1988, S. 55-61.

Gahama, Joseph, Le Burundi sous administration belge, Paris 1983, passim.

Vgl. Chrétien, J.-P., Féodalité ou féodalisation du Burundi sous le Mandat belge, Paris 1982, S. $367 \mathrm{ff}$. 
Burundi und Ruanda von Belgien führten. ${ }^{13}$ Gemäß der Provisorischen Verfassung vom 26. November 1961 entstand ein konstitutionelles (Tutsi-) Königreich unter Mwami (König) Mwambuts IV. (bestätigt durch die Verfassung (Verf) vom 16. Oktober 1962).

Zum besseren Verständnis der aktuellen Probleme (auch) im Rechts- und Gerichtsbereich folgen hier wichtige Ereignisse im unabhängigen Burundi in Kurzform:

1965: Blutiger Putschversuch von Hutuoffizieren der Armee - vom König und (Tutsi-) Hauptmann Michel Micombero hart niedergeschlagen.

1966: Kronprinz Charles Ndizeye folgt seinem Vater als König Ntare IV., suspendiert die Verf, wird gestürzt von Premierminister Micombero, der am 18.11.1966 die Republik ausruft und die Einheitspartei Union pour le progrès national (UPRONA) etabliert. ${ }^{14}$

1971 Hutu-Staatsstreich-Versuch.

1972 Rückkehr von König Ntare IV. aus dem Exil, blutige Massaker an den Hutu (100-200000 Tote und ca. 200000 Hutuflüchtlinge).

11.7.1974 neue Verf, die die Position Micomberos stärkt.

1976 Oberstleutnant Jean-Baptiste Bagaza ${ }^{15}$ (Tutsi) stürzt in unblutigem Staatsstreich Micombero, der sich zwischenzeitlich selbst zum Generalleutnant befördert hatte. UPRONA wiederbelebt.

18.11.1981 Verf der 2. Republik durch Referendum angenommen. Diktatur Bagazas.

3.9.1987 Unblutiger Sturz Bagazas durch Major Pierre Buyoya (Tutsi); Einsetzung des 31 köpfigen Comité militaire pour le salut national (CMSN).

1988 Hutu töten etwa 3000 Tutsi-Hima, die tutsibeherrschten Streitkräfte daraufhin etwa 2000 Hutu; bei den folgenden Unruhen sterben mehr als 120000 Burunder. Buyoya ernennt Adrien Sibomana (Tutsi, der mit einer Hutu verheiratet ist) zum Premierminister.

5.2.1991 Charte de l'unité nationale (CUN) wird durch Referendum angenommen.

9.3.1992 Verf der 3. Republik wird durch Referendum angenommen.

2.6.1993 Melchior Ndadaye (Hutu) (Front démocratique du Burundi [FRODEBU]) wird zum Präsidenten der Republik gewählt.

21.10.1993: Der Putschversuch der tutsibeherrschten Armee führt zur Ermordung von Ndadaye und sechs führenden FRODEBU-Politikern, scheitert aber. ${ }^{16}$ Da-

Gahama, Joseph, Les partis politiques et la recherche de l'indépendance au Burundi, in : Nsabimana (Hrsg.) : Histoire sociale de l'Afrique de l'Est (XIXe- XXe siècle ), Paris 1991, S. 135 ff.

14 Dazu näher: Manirakiza, Marc, Burundi: De la révolution au régionalisme 1966-1976, Brüssel 1992, passim.

15 Dazu näher: Manirakiza, Marc, Burundi: La déviance d'un pouvoir solitaire, Le régime Bagaza 1976-1987, Paris Brüssel 1997, passim.

16

Zum Umfeld vgl. z.B. Kühne, Winrich/Mair, Stefan, Der Konflikt an Ostafrikas Großen Seen und der Machtwechsel in Zaire, in: Jahrbuch Dritte Welt 1998, S. 132-165. 
nach und in den folgenden Monaten töten Hutu ca. 100000 Tutsi. ${ }^{17}$ Ausbruch des Bürgerkriegs. Mehr als 200000 Flüchtlinge gehen nach Tansania, Zaire, Europa etc., 600000 Binnen-Flüchtlinge.

1994 Nach sechsmonatiger Interimsherrschaft des Hutu Sylvestre Ntibantunganya (FRODEBU) einigen sich FRODEBU und UPRONA auf eine neue Machtverteilung: Cyprien Ntarayamira (FRODEBU) wird Präsident, Anatole Kanyenkiko (UPRONA) Premiernminister.

6.4.1994 Ntarayamira kommt mit dem ruandischen (Hutu-) Diktator Juvénal Habyarimana in Kigali (Ruanda) bei einem ungeklärten Flugzeugabsturz ums Leben. Ntibantunganya wird Präsident, Antoine Nduwayo (UPRONA) Premierminister.

1996 scheitert der Einsatz einer internationalen Truppe der UNO am Widerstand der Armeeführung.

25.7.1996 Putsch der Armee und Rückkehr Buyoyas. Vizepräsident wird Domitien Ndayizeye (Hutu). Im Übergangsparlament (ab 18.7.1998) haben von 121

Sitzen die FRODEBU 65, die UPRONA 16, Sonstige 13, Unabhängige 27 Sitze inne.

\section{Staatliches Gerichtswesen in Burundi}

Die Übergangsverfassung vom 28. Oktober 2001 (loi no. 1/017) ${ }^{18}$ (ÜVerf) regelt die Judikative im VIII. Abschnitt und garantiert die Unabhängigkeit und die Unparteilichkeit der Justiz sowie den Grundsatz der Öffentlichkeit von Gerichtsverhandlungen und Urteilsverkündung. Das Friedens- und Versöhnungsabkommen von Aruscha ${ }^{19}$ vom 28. August 2000 enthält in seinem 2. Protokoll Verfassungsgrundsätze, u.a."Demokratie und gute Regierungsführung" mit Regelungen zur Reform und Modernisierung des Justizsektors. ${ }^{20}$

Näher: Associations de la Société civile, Burundi : Génocide d'octobre 1993, Bujumbura 1996.

Die Übergangsverfassung ersetzt den Übergangsverfassungsakt für die Republik Burundi (Acte constitutionnel de transition de la République du Burundi) vom 6. Juni 1998 (Gesetzblatt von Burundi (Bulletin Officiel du Burundi) Nr. 7/98, S. 469-483 vom 1. Juli 1998), der seinerseits die Verfassung der Republik Burundi vom 13. März 1992 (Gesetzblatt Nr. 4/92, S. 95-155 vom 1. April 1992) und die Gesetzesvertretende Verordnung (Décret-loi) Nr. 1/001/96 vom 13. September 1996 über die Organisation der Übergangseinrichtungen (Organisation du Système institutionnel de Transition) aufhob.

Accord d'Arusha pour la Paix et la Réconciliation au Burundi.

Einige zusätzliche Regelungen dazu finden sich auch im 1. Protokoll zum Friedens- und Versöhnungsabkommen: "Natur des Konflikts in Burundi, Genozid und Ausschluss sowie ihre Lösungen”, z.B. zur Förderung einer unparteilichen und unabhängigen Justiz. 
In der besonderen Lage von BU kommt Art. 166 ÜVerf große Bedeutung zu, der anordnet: "Bei Einstellungsverfahren und Ernennungen im Justizbereich sind die Verbesserung des ethnischen Gleichgewichts und der Gleichberechtigung der Geschlechter zu beachten.”

\subsection{Gerichte und Staatsanwaltschaften}

Burundi verfügt über ein relativ ausgebautes Gerichtssystem: ${ }^{22}$

- Das Verfassungsgericht (Cour constitutionnelle) (vgl. Art. 180ff. ÜVerf) soll die Einhaltung der Normen der ÜVerf, besonders der Grundrechte und Grundpflichten (3. Abschnitt, Art. 15 - 64 ÜVerf) garantieren.

- Der Oberste Gerichtshof (Cour suprême), ist die höchste Instanz in Zivil-, Straf- und Verwaltungssachen. $^{23}$

- Der Hohe Gerichtshof (Haute Cour de justice) (vgl. Art. 188 ff. ÜVerf) ist nur für Anklagen gegen den (Staats-)Präsidenten, den Vizepräsidenten, den Nationalversammlungspräsidenten und den Senatspräsidenten zuständig.

- Drei Berufungsgerichte (Cours d'appel) ${ }^{24}$ sind vergleichbar mit Oberlandesgerichten und entscheiden über Berufungen gegen Entscheidungen der Landgerichte (Tribunaux de grande instance), der Arbeits- und der Handelsgerichte und in erster Instanz (Chambres criminelles) über schwerste Verbrechen, die nicht von/an Soldaten, für die Militärstrafgerichte zuständig sind, begangen wurden. ${ }^{25}$

- 17 Landgerichte (Tribunaux de grande instance) ${ }^{26}$ sind in jeder der 17 Provinzen des Landes etabliert. Sie sind primär Berufungsgerichte gegen Entscheidungen der Amtsgerichte (Tribunaux de résidence); sie haben aber auch eine erstinstanzliche Auffangzu-

In der Praxis dürfen Richter und Staatsanwälte daraufhin nur auf Vorschlag des Oberen Justizrats (Conseil supérieur de la Magistrature), dem Tutsi- und Hutujuristen angehören, und mit Zustimmung des Senats (Art. 141 ÜVerf), dem Vertreter der Provinzen angehören, ernannt werden.

Grundsätzlich geregelt im Code de l'organisation et de la compétence judiciaire (Gerichtsverfassungsgesetz) i.d.F. vom 14. Januar 1987. Abramowski, Jasper / Nzeyimana, Laurent / Ntambwiriza, Zacharie / Nshimirimana, Dona Fabiola, Étude sur le secteur judiciaire : Atouts et faiblesses du Système judiciaire du Burundi, Bujumbura 2001, passim m.w.N., haben viele Fakten und Zahlen zum Justizsektor zusammengestellt und kommentiert; darauf kann hier - teilweise nach Überprüfung und Aktualisierung - zurückgegriffen werden.

Er ist besetzt mit 10 Richtern und gliedert sich in die Chambre de Cassation, Chambre administrative und Chambre judiciaire.

Ein viertes Oberlandesgericht soll in Bururi eingerichtet werden; für seine Einrichtung fehlen jedoch (noch) die Mittel.

Die drei Berufungsgerichte sind insgesamt mit 25 Richtern besetzt. Die Chambres criminelles entscheiden in der Zusammensetzung mit einem Berufsrichter als Vorsitzendem und vier ehrenamtlichen (Laien-) Richtern (Beisitzern = assesseurs).

Ein weiteres Landgericht soll in Rumonge eingerichtet werden; für seine Errichtung fehlen jedoch (noch) die Mittel. 
ständigkeit in Zivil- und Strafsachen, d.h. sie sind zuständig, falls keine andere Zuständigkeit gesetzlich ausdrücklich festgelegt wurde. ${ }^{27}$

- 125 Amtsgerichten vergleichbare Tribunaux de résidence wurden in jeder Gemeinde des Landes errichtet. ${ }^{28}$ Zivilrechtlich sind sie zuständig bis zu einem Streitwert von von Francs Bu umgerechnet - 400 Euro bzw. unabhängig vom Streitwert in Ehe-, Familien-, Erbschafts- und Landstreitigkeiten. In Strafsachen können sie Freiheitsstrafen bis zu zwei Jahre verhängen. Da in Amtsgerichtsverfahren kein Staatsanwalt anwesend ist, übernehmen die Richter auch die Funktion der Staatsanwaltschaft und sind insoweit weisungsgebunden. Das ist rechtsstaatlich hoch bedenklich und wird noch dadurch gravierender, dass etwa 20\% der Amtsrichter keine volle juristische Ausbildung durchliefen.

- Zwei Arbeitsgerichte (Tribunaux de travail) wurden in Bujumbura und in Gitega errichtet,ein Handelsgericht (Tribunal de commerce) in Bujumbura.

- Zwei Verwaltungsgerichte (Cours administratives) bestehen in Bujumbura und Gitega.

Die Staatsanwaltschaft ist wie folgt gegliedert: Die Generalstaatsanwaltschaft der Republik (Parquet général de la République), deren Procureur général de la République beim Obersten Gerichtshof auftritt, ist Vorgesetzte der Generalstaatsanwälte (Procureur général près de la Cour) bei den Oberlandesgerichten. In jedem Landgerichtsbezirk sind zwei bis drei Staatsanwälte (Procureurs) für die Staatsanwaltschaft (Parquet) tätig.

Die 78 Rechtsanwälte des Landes sind alle in Bujumbura niedergelassen.

Die Polizeiorganisation in BU ist vielfältig und verwirrend; das ist möglicherweise mit Machtdenken der Regierenden zu erklären. Dem Justizministerium und damit der Staatsanwaltschaft untersteht nur die Justizpolizei (Police judiciaire des parquets - PJP) mit 126 Beamten, die auf ein Generalkommissariat, 17 Kommissariate und 10 Polizeiwachen aufgeteilt sind. $^{29}$

Die Militärstrafgerichtsbarkeit besteht aus dem Militärgerichtshof (Cour militaire), gegen dessen Urteile im Wege der Revision die Chambre de cassation des Obersten Gerichtshofs angerufen werden kann, und den Kriegsgerichten (Conseils de guerre) für die Ahndung von Straftaten von Soldaten unterhalb des Majorsrangs. Rechtsmittel gegen Entscheidungen der

Insgesamt sind an den Landgerichten 80 Richter tätig, die wie die Richter an den vorstehend genannten Gerichten Volljuristen mit juristischem Hochschulabschluss sind. Sie entscheiden in Chambres civiles und Chambres pénales.

Insgesamt sind bei den Amtsgerichten 547 Richter tätig, die in Spruchkörpern mit einem Berufsrichter und zwei ehrenamtlichen (Laien-) Richtern (Beisitzern = assesseurs) entscheiden.

29

Daneben gibt es die Gendarmerie, die Police de la sécurité publique, die Police de l'air, des frontières et des étrangers und die Police de la documentation nationale. 
Conseils de guerre gehen an die Cour militaire, die erstinstanzlich für die Ahndung von Straftaten von Oberstleutnanten und höherrangigen Offizieren zuständig ist. Unter Aufsicht des Generalstaatsanwalts der Republik arbeiten bei den Militärgerichten Militärstaatsanwälte (Auditeurs militaires).

In Burundi gibt es 11 Justizvollzugsanstalten, die größtenteils in der ersten Hälfte des vergangenen Jahrhunderts gebaut wurden. Auf eine Kapazität von 3650 Häftlingen angelegt, mussten sie 9042 Gefangene aufnehmen. ${ }^{30}$ Das Gefängnispersonal beläuft sich auf 750 Beamte und Angestellte (einschließlich des Verwaltungspersonals).

\subsection{Probleme der burundischen Justiz}

Obwohl die burundische Justiz im afrikanischen Rahmen und "auf dem Papier" als relativ entwickelt und gut organisiert erscheint, obwohl sie von einem dem Mehrheitsvolk der Hutu angehörenden Minister, Fulgence Dwima Bakana, geleitet wird, ${ }^{31}$ hat sie große Probleme:

Viele Gesetze stammen noch aus der belgischen Mandatszeit und müssten reformiert werden. Für die Reform fehlen aber die personellen und sachlichen Mittel - der gesamte Haushalt des Justizministeriums betrug im Jahre 2001 rund 3,7 Mio Euro, d.h. etwa 3\% des nationalen Budgets - und teilweise der notwendige politische Wille. Die Beförderungschancen für Justizangehörige sind gering. Viele, oft dynamische und qualifizierte Justizmitarbeiter quittieren den wenig lukrativen Dienst und suchen Arbeit im privaten Sektor (brain drain). Immerhin wurden 1999 das Staatsangehörigkeitsgesetz (Code de la nationalité), das Beamten- und Richtergesetz (Code des magistrats) und die Strafprozeßordnung (Code de Procédure pénale) erneuert. Daneben wenden die staatlichen Gerichte Gewohnheitsrecht an. $^{32}$

Und am 9. März 1999 verkündete das Justizministerium den "Plan zur Reform und Modernisierung der burundischen Rechtspflege und des burundischen Strafvollzugs" ("Plan de réforme et de modernisation du système judiciaire et pénitentiaire burundais") und versucht ihn - einschließlich der Verbesserung der Justizorganisation - seither in der Praxis umzusetzen.

30

31

32

Stichtag: 30. September 2001.

Von den 26 Ministern der am 1. November 2001 eingesetzten Übergangsregierung sind 14 Hutu und 12 Tutsi.

Zum Rechtspluralismus in afrikanischen Ländern vgl. die sorgfältig differenzierte Zusammenfassung von Sippel, Harald / Wanitzek, Ulrike, Recht, in: Das kleine Afrika-Lexikon, Stuttgart 2002, S. 161-165. 
Das geringe Ansehen der burundischen Justiz in der Bevölkerung beruht auf verbreiteter Korruption im Justizapparat ${ }^{33}$, auf Kritik an der Langsamkeit der Justiz und fehlerhafter Rechtsanwendung. Die Langsamkeit beruht ihrerseits auf unzureichender personeller und materieller Ausstattung von Gerichten, ${ }^{34}$ Staatsanwaltschaften und Justizvollzugsanstalten. Davon konnte sich der Verfasser in Bujumbura und in Gitega bei verschiedenen Stichproben selbst überzeugen; ${ }^{35}$ auch das bereits angeführte Normendefizit und die unzulängliche Publikation von - teilweise veralteten und lückenhaften - Gesetzen und Verordnungen tragen dazu bei. Bürgerkriegsbedingte Hemmnisse vor allem in einigen speziell betroffenen Provinzen vergrößern die Probleme. Die Aufarbeitung der seit 1993 im Bürgerkrieg begangen Verbrechen leidet ebenfalls, so dass bereits von einer "Kultur der Straflosigkeit" gesprochen wird. ${ }^{36}$ Da es z.B. nur drei (Große) Strafkammern, d.h. je eine bei den Oberlandesgerichten gibt, die sich mangels funktionierender DienstKfz grundsätzlich auch nicht zu Verhandlungen aufs Land begeben können, haben Zeugen und Nebenkläger oft Schwierigkeiten bei Gericht zu erscheinen. ${ }^{37}$ Viele Beschuldigte beklagen sich über schlechte Behandlung durch die Polizei. Die Rückkehr von Flüchtlingen, etwa aus der Vereinigten Republik Tansania und der Demokratischen Republik Kongo, die z.B. ihr Grundeigentum zurückverlangen, belastet die Justiz zusätzlich. Auch die fehlende Veröffentlichung von höchstrichterlichen Entscheidungen, ${ }^{38}$ fehlende Fachliteratur und viel zu geringe Fort- und Weiterbildungsmöglichkeiten für das Justizpersonal erschweren gerechte und korrekte

Die Bezahlung der Richter, Staatsanwälte, Rechtspfleger etc. ist niedrig und erfolgt nicht regelmäßig. Ein Amtsrichter verdient etwa 50, ein Richter an der Cour suprême etwa 90 Euro im Monat.

Beim Oberlandesgericht Gitega gingen im Jahre 2000 z.B. 501 neue Vorgänge ein zu denen noch 248 aus dem Vorjahr anhängige kamen. Erledigt werden konnten im Jahre 2000 nur 170 Sachen, so dass der Überhang sich am Jahresende praktisch verdoppelt hatte.

Beim Landgericht Gitega fehlte es z.B. beim (unangekündigten) Arbeitsbesuch des Verfassers am 28. August 2002 sogar an Papier, um die Urteile auf den mechanischen Schreibmaschinen zu schreiben.

Das beginnt bereits bei den Ermittlungen: Die Police judiciaire des parquets (PJP) verfügt landesweit nur über ein funktionierendes Kfz, für das sie 50 Liter Treibstoff im Monat erhält.

Teilweise haben es Nichtregierungsorganisationen wie die Ligue burundaise des droits de l'homme - ITEKA übernommen, Zeugen und Opfer zu Gerichtsorten zu fahren. Zur internationalen Kooperation im Bereich der Justiz näher: Accad, Lucien, Rapport de la Mission d'étude sur le droit et la justice au Burundi, Bujumbura 2002, S. 36-74.

Die International Human Rights Law Group versucht seit 2002 mit der Veröffentlichung von kommentierten Entscheidungen zu speziellen Themenbereichen, z.B. Frauen und Erbgang, in ihrer Revue de droit et de jurisprudence zu helfen. Aber diese gut gemeinten eklektischen Hefte können keine Amtlichen Sammlungen ersetzen. Auch die von der ITEKA herausgegebenen "Bulletins d'information" erfüllen nicht die notwendigen Anforderungen an eine systematische Veröffentlichung höchstrichterlicher Entscheidungen. 
Rechtsfindung. Mängel der Juristenausbildung ${ }^{39}$, zu geringe Repräsentanz von Hutu ${ }^{40}$ und Frauen $^{41}$ im Justizapparat sind evident.

Bei aller Kritik scheint es aber bemerkenswert, wie viele Angehörige der burundischen staatlichen Justiz sich tagtäglich, allen widrigen Umständen zum Trotz aktiv und pflichtgetreu um Recht und Gerechtigkeit bemühen. Und ihr Beitrag zu (Rechts-) Frieden und Versöhnung muss gebührend geachtet werden.

Mehrere multi- und bilaterale Nichtregierungsorganisationen (NRO) helfen ihnen beim (Wieder-) Aufbau eines Rechtsstaats in BU. Die Deutsche Gesellschaft für Technische Zusammenarbeit (GTZ) plant ebenfalls ein Projekt zur Justizförderung in Burundi. ${ }^{42}$

\section{Bashingantahe}

Es gilt, einen Ausweg aus den bereits kurz nach Erlangung der Unabhängigkeit eskalierten und bis heute fortdauernden Spannungen zwischen Hutu und Tutsi und - über eine Analyse der Wurzeln der Entzweiung - hin zu einer fortschreitenden Aussöhnung zu finden. Dafür empfehlen die Nationale Kommission zum Studium der Frage der Nationalen Einheit, burundische Juristen und Politiker, ja sogar Theologen ${ }^{43}$ die Rehabilitierung / Reaktualisierung / Revitalisierung der Werte und der Einrichtung der Bashingantahe (ibanga ry'abashingantahe) ${ }^{44}$ als "Bashingantahe neuer Darstellungsweise" (Bashingantahe nouveau style), d.h. als einheitliches, solidarisches (Richter-) Korps - genannt Urwego

Das Studium der Rechte ist in Burundi an den Rechtsfakultäten der Universität von Burundi und der (privaten) Universität von Ngozi möglich; viele burundische Juristen haben aber ganz oder teilweise im Ausland, vor allem in Belgien, studiert.

Offizielle Statistiken dazu gibt es nicht. Die Nichtregierungsorganisation Avocats sans frontières (AsF) schätzt, dass bei den höheren Gerichten etwa 90\% der Richter Tutsi sind, während bei den Amtsgerichten fast nur Hutu tätig sind.

Der Anteil der Richterinnen beträgt bei den höheren Gerichten 21\%, bei den Amtsgerichten $24 \%$.

Die GTZ hat bereits in den Jahren 1991 ff. Gründung und Arbeit des staatlichen burundischen Menschenrechtszentrums unterstützt. Dieses Zentrum heißt heute Centre de promotion des droits de la personne humaine et de prévention du génocide und ressortiert zum Justizministerium.

Grundlegend und m.w.N.: Ntabona, Adrien, Itineraire de la sagesse - Les Bashingantahe hier, aujourd'hui et demain au Burundi, Bujumbura 1999, passim, besonders S. $212 \mathrm{ff}$.

Im Auftrag der burundischen Regierung haben Ntahombaye, Phillipe / Ntabona, Adrien / Gahama, Joseph / Kagabo, Liboire (Hrsg.), The Bashingantahe institution in Burundi - A Plurisisciplinary Study, Bujumbura 1999, die Geschichte der Bashingantahe und die Möglichkeiten ihrer Revitalisierung näher untersucht (m.w.N.). 
rw'Abashingantahe -, ${ }^{45}$ mit gesellschaftlichem, politischem und rechtlichem Auftrag. Sie sollen:

- durch Schlichtung oder Urteil Streitigkeiten gemäß dem Stand der jeweiligen Konflikte befrieden,

- Personen und Familien untereinander aussöhnen,

- Verträge beurkunden (z.B. Eheverträge, Erbverträge und/oder Testamente, Kaufverträge, Schenkungen),

- über Wahrheit und Gerechtigkeit in ihrem Ort wachen,

- Sicherheit von Personen und Sachen in ihrer Umgebung gewährleisten,

- politische Mächte auf allen Stufen beraten und ausgleichen und

- zugunsten des Gemeinwohls und der Grundrechte bei Bedarf sprechen. ${ }^{46}$

In Gitega kamen Bashingantahe im Februar und im April 2002 zum von den Bashingantahe der 17 Provinzen des Landes gewählten 17köpfigen "Nationalrat der Bashingantahe" ("Conseil national des Bashingantahe") zusammen und nahmen am 5. April 2002 die Charta der Bashingantahe (Charte des Bashingantahe) an, das erste schriftliche Dokument seit der Entstehung der Einrichtung am Ende des 17. Jahrhunderts. Es enthält in $11 \mathrm{Ab}-$ schnitten u.a. Hinweise und Regeln zu Kriterien (Voraussetzungen), Aufgaben, Bestellung und Platz der Bashingantahe im System von Recht, Verwaltung und Politik in BU, Sanktionen bei Fehlverhalten eines Mushingantahe sowie zum Finanzwesen der Bashingantahe. Die Frage, ob es sich in einem oralen System dabei nicht um einen Systembruch handelt, muss dabei wohl hinter dem Gewinn an Rechtsstaatlichkeit - Vertrauensschutz, Bestimmtheit, Bestimmbarkeit - zurücktreten.

Während einige allerdings den Bashingantahe vorwerfen, ihre Pflichten nur unvollkommen wahrgenommen zu haben, bescheinigen ihnen andere, als Pfeiler der gesellschaftlichen Ordnung während der "Krise" und Vertriebenen beigetragen zu haben.

\subsection{Begriff Bashingantahe}

Im Singular bedeutet mushingantahe auf Kirundi wörtlich "Friedensrichter" oder "Schiedsrichter und Erzeuger von Gerechtigkeit" und bezeichnet im sozio-kulturellen Sinn: eine reife Persönlichkeit mit Wahrheitssinn, hoher Intelligenz, Fleiß, Selbstvertrauen sowie Gerechtigkeits- und persönlichem, familiärem und sozialem Verantwortungssinn, bezeichnet also einen ehrenhaften, erfahrenen, weisen, selbstbeherrschten, ausgeglichenen, kom-

Vgl. dazu Ntabona, Adrien, L'institution des Bashingantahe et la modernité, Bujumbura 2002, passim und besonders S. $12 \mathrm{ff}$.

Ntabona a.a.O. FN 45, S. 13.

47

Gemeint sind die Bürgerkriegsereignisse seit 1993. 
promissbereiten, moderaten, im Ausdruck starken aber verbal maßvollen ${ }^{48}$ (ijambo) Mann, kurz, die burundische Variante eines Gentleman ${ }^{49}$. Traditionelle Ausbildung und Initiation junger Männer soll dazu beitragen, sie zu solcher gesellschaftlichen Reife als verantwortungsvolle Persönlichkeiten (ubushingantahe) zu befähigen. 50

Im Rechtssinn waren bashingantahe (Plural) Anwender von Gewohnheitsrecht (traditionellem Recht) ${ }^{51}$ mit der Aufgabe, Konflikte zu untersuchen, mündlich zu verhandeln und zu schlichten. Die hinter ihrem Denken und Handeln stehende Idee war auf schiedsrichterlich-vermittelnden Ausgleich und Versöhnung, nicht auf Repression gerichtet.

So sicherten die Bashingantahe Gewaltenteilung und Ordnung. Der burundische Autor Emil Mworoha formulierte ${ }^{52}$ : Die Bashingantahe bildeten "eine Institution, die die gesamte politische und administrative Struktur von der Basis bis zur Spitze der staatlichen Hierarchie durchdrang". In einer sakral bestimmten traditionellen Monarchie an der Spitze des Staates, der Gesellschaftsstruktur und der pyramidal aufgebauten, vom Königshof abhängigen Verwaltung waren die Bashingantahe etabliert als Repräsentanten des Denkens und Trachtens, das von den Wurzeln der Gesellschaft ausging, hatten also ein loyales Wächteramt (in) der Gesellschaft inne. ${ }^{53}$

\subsection{Geschichte der Bashingantahe}

Ein Kirundi-Sprichwort sagt: Kein König kann ohne Unterstützung der Bashingantahe regieren; ein Prinz, der nicht auf die weisen Ratschläge der Bashingantahe hört, darf nicht zum König gekrönt werden (Umwami agirwa n'abagabo; kananira abagabo ntiyimye). Das deutet bereits darauf hin, dass die Einrichtung der Bashingantahe so alt ist wie die burundi-

Sprache und Kultur (auch: Rechtskultur) sind speziell in Afrika (aber nicht nur dort) miteinander verwoben. So verfolgt auch der mushingantahe in/mit seinen Reden gesellschaftliche, pädagogische/belehrende, politische, ethische und teilweise sogar wissenschaftliche (Überlieferungs-) Ziele, näher: Nizigiyimana, Domitien, Tehe decency of speech as an enhancing factor fort he bashingantahe institution, in: Ntahombaye (u.a.) op. cit. FN 44, S. 143-153 (149 ff.).

Näher: Ngorwanubusa, Juvénal, The bashingantahe institution and the universal ideal of a gentleman, in: Ntahombaye (u.a.) op. cit. FN 44, S. 154-163, passim.

Den Begriff intahe als kulturellen Wert, der auf Kirundi - verkürzt ausgedrückt - Kraft für Frieden und Glück beinhaltet, untersucht Bigirumwami, Joseph, Use of the word intahe and ist corollaries in Burundian language and culture, in: Ntahombaye (u.a.) op. cit. FN 44, S. 56 ff. Dazu näher : Sippel/Wanitzek, Recht op. cit. FN 32, S. 162 ff.

L'enracinement de la recherche historique dans la culture populaire de «l'Enclos » et de « la colline » au Burundi du XIXème siècle, in: Culture et Société -Revue de Civilisation burundaise Nr. 11, S. 12.

53 Ntabona, Adrien, Responsabilité sociale et tissage de l'unité des Barundis, in: Au cœur de l'Afrique Band LX Nr. 2, Paris 1990, S. 147. 
sche Monarchie. Sie wurde in der Tat vom ersten König, Ntare Rushatsi, Ende des 17. Jahrhunderts eingesetzt. ${ }^{54}$

Und noch heute enthält ein neu kooptierter Mushingantahe bei der Zeremonie den symbolischen (Richter-) Stock mit den Worten überreicht: "Dies ist der Stock der Gerechtigkeit, der Deinem Vater und Deinem Großvater von König Ntare Rushatsi verliehen wurde.”

Als Bashingantahe wurden Tutsi- und Hutumänner bestellt. ${ }^{55}$ Vor der Bestellung als Mitglied der Bashingantahe musste der Kandidat folgende Voraussetzungen erfüllen: ${ }^{56}$ Er musste mindestens 25 Jahre $^{57}$ alt und verheiratet sein, furchtlos die Wahrheit sagen (andernfalls konnte ihm "der Stock" auch später wieder aberkannt werden), Sinn für Ehre und Würde haben. Um "Käuflichkeit" von Urteilen zu vermeiden, wurde vom Kandidaten ein gewisser Wohlstand verlangt. ${ }^{58}$

Nachdem ein Mann, dessen Qualifikation aufgefallen war, unter seinesgleichen größere Mengen Bier verteilt hatte, wurde er als Aspirant (bakungu b'imbere) bestellt und sein Vater konnte seine Kooptation beantragen. Falls die Ältesten ihn akzeptierten, nahm er als Kandidat (umunyamutamana oder umunyampaya) angeleitet von einem Paten (umuhetsi) an einer mehrjährigen Initiationsphase teil. Die Bestellung als mushingantahe fand auf einem großen Fest statt. Der Pate verkündete dabei: "Von jetzt an ist X ein mushingantahe, vom König und den Bashingantahe bestellt ist er das Auge des Königs; zögert nicht, ihm Eure Probleme anzuvertrauen." Vor der Ablegung des Eids fragte der Zeremonienmeister den "Neuen": "Akzeptierst Du, nach den Gesetzen dieses Landes zu leben, barmherzig zu sein und dem Bedürftigen zu helfen? Akzeptierst Du das Land zu erleuchten, Gerechtigkeit adäquat zu gewähren und Korruption beim Richten zu vermeiden?" Die Antwort des zwischen seiner Frau und seinem Paten Stehenden war der folgende Amtseid: "Ich akzeptiere und gelobe vor dem König und den Bashingantahe stets in den Konflikten, die ich zu ent-

Gahama, in: Ntahombaye (u.a.) op. cit. FN 44, S. 23.

Die Twamänner wurden von Anfang an von diesem Richteramt ausgeschlossen, weil es ihnen angeblich an "common sense" fehlte (vielleicht auch weil sie einer vielfach unterdrückten Minderheit angehörten - d.Verf.).

Zum Folgenden näher: Ntabona, Adrien, The bashingantahe institution: from tradition to modernity, in: Ntahombaye (u.a.) op. cit. FN 44, S. 40 (41 ff.).

Das war gemessen an der damals geringen durchschnittlichen Lebenserwartung bereits ein stattliches Alter, das Reife garantieren sollte. Bei einer durchschnittlichen Lebenserwartung von 42 Jahren verwundert es nicht, dass in Burundi - und insgesamt in Afrika - ein alter Mann schon deswegen respektiert wird, weil er so stark war, trotz aller widrigen Umstände so alt zu werden, d.h. zu überleben.

Anders gewendet: Arme und Bettler konnten nicht kooptiert werden. 
scheiden habe, der Wahrheit die Ehre zu geben." 59 Nach einer Ansprache des Vertreters der Bashingantahe, in der er auf seine Pflichten hingewiesen wurde, lud dieser ihn ein, auf seinem Gerichtsstuhl Platz zu nehmen und verlieh ihm den (Richter-) Stock (intahe) als Symbol der Autorität und Grundlage seiner Machtausübung, kurz: neuer sozialer Identität; dieser 50 bis $150 \mathrm{~cm}$ lange Stock diente auch als Stütze für ältere Richter (kwishimikiza inkoni -sich auf einen Stock stützen) und gab psychokinetisch ein Gefühl der Stabilität und Sicherheit.

Danach folgten Glückwünsche und Lobgesänge der Frauen auf den neuen Richter. Dieser genoss fortan großen Respekt in der Gemeinschaft. ${ }^{60}$

Die Bashingantahe hatten drei Arten von Verpflichtungen:

- Moralische Verpflichtungen: Sie hatten Vorbildfunktion in der Gesellschaft als "Kornspeicher voll von Frieden".

- Soziale Verpflichtungen: Neben der Streitregulierung mit der Friedensfunktion waren sie zum Schutz der Schwachen da.

- Politische Verpflichtungen: Bei Pflichtverletzungen wurden er und seine Familie geächtet ("gesellschaftlicher Tod"), durften z.B. kein Wasser mehr aus dem Gemeinschaftsbrunnen schöpfen (kumucira ivomo). Nur ganz ausnahmsweise waren nach einer langen Reuephase eine Reinigung und ein neuer Amtseid zulässig. Da die Bashingantahe den Eid also nicht brachen (jedenfalls intensiv versuchten, ihn nicht zu brechen) suchten der König und andere Staatsautoritäten unter den besonders bekannten Bashingantahe ihre Ratgeber auch für die (nichtrichterlichen) Staatsgeschäfte.

Während die deutsche Schutzgebietsverwaltung Institution und Tätigkeit der Bashingantahe im Wesentlichen unangetastet ließ, beschloss die belgische Mandatsverwaltung ab 1921 deren Entscheidungen zu überprüfen und "unangemessene" Urteile abzuändern. Damit beraubte sie die Bashingantahe ihrer Autorität und machte sich zum Garanten des Gewohnheitsrechts und seiner praktischen Anwendung. Die Missionare versuchten jetzt, die einheimischen Christen ganz dem Einfluss der Bashingantahe zu entziehen.

Es ist auch eine modifizierte Eidesformel überliefert: "Ich nehme die Verpflichtung an. Falls ich sie nicht erfülle, soll sie meinen Schädel zerschmettern. Ich verpflichte mich, alle Konfliktbeladenen zu versöhnen, Waisen und Witwen zu verteidigen, die Wahrheit zu sagen, die durch das Feuer geht ohne zu brennen, und das Übel fortzuweisen." (zitiert bei Rutake, Pascal, in: Ntahombaye (u.a.) op. cit. FN 44, S. 77 nach: Hakizimana, I., L'institution des Bashingantahe au Burundi, Bujumbura 1976).

60

Wenn jemand einen Prozess verlor, sagte er z.B.: "Mit dem Urteil bin ich nicht einverstanden, aber mit dem Bashingantahe bin ich einverstanden!" 
Mit dem Gesetz vom 5. Oktober 1943 schaffte die Mandatsmacht die traditionelle Schiedsgerichtsbarkeit auf allen Ebenen ab und ersetzte sie durch Gewohnheitsrechts-Gerichte auf drei Ebenen:

In den Häuptlingsgerichten durften Bashingantahe nur beratend mitwirken; vom mushingantahe wurden ihre Richter zu mucamanza (kolonialregierungsangestellten Beisitzern) degradiert.

In den Distriksgerichten, deren Vorsitz der (belgische) Distriktskommissar innehatte, waren sie gar nicht vertreten; Beisitzer waren dort vom König bestellte Häuptlinge (Baganwa).

Das Königliche Gericht wurde von der Kolonialverwaltung besetzt.

Im Vordergrund bei der Besetzung dieser Posten standen nicht mehr die BashingantaheVoraussetzungen/-Pflichten sondern nur noch Intelligenz und Kollaborationswille mit den Kolonialbehörden.

Nach der Erlangung der Unabhängigkeit (1. Juli 1962) wurden zwar Bashingantahe wieder eingerichtet; jetzt stand jedoch dabei der folkloristische Aspekt im Vordergrund. Da landesweit große Festivitäten für ihre Investitur organisiert werden sollten (und wurden), war für die Auswahl der Kandidaten deren Reichtum wichtigstes Kriterium. Unter der zweiten Republik, d.h. nach dem 18. November 1981, wurden die Bashingantahe (wieder) ganz abgeschafft.

\section{Aktuelle Situation}

Die ehrenamtlichen Schiedsgerichte / Schlichtungsstellen ${ }^{61}$ der Bashingantahe üben heute in BU auf der Basis von Gewohnheitsrecht lokal in öffentlichen mündlichen Verhandlun-

Auch in Nordrhein-Westfalen (und in einigen anderen Ländern der Bundesrepublik Deutschland in der Nachfolge Preußens) haben ehrenamtliche Schiedsstellen / Schiedsämter / Schiedspersonen, deren Existenz einem demokratisch-sozialen Bedürfnis zu entsprechen scheint, eine lange Tradition. So organisierten in Bielefeld die 13 ehrenamtlichen, von den Bezirksvertretungen gewählten Schiedspersonen am 22. März 2003 die 175-Jahrfeier der Einrichtung. Sie streben in Sühneversuchen/-verfahren in nachbarrechtlichen und Ehrschutzangelegenheiten, bei fahrlässigen oder leichten Körperverletzungen, Sachbeschädigungen, Hausfriedensbrüchen, Verletzungen des Briefgeheimnisses und Vermögensstreitigkeiten bis zu 600 Euro eine unbürokratische, gütliche Einigung / Streitvermittlung bzw. einen Täter-/Opferausgleich an und helfen so, teuere gerichtliche Verfahren zu vermeiden. (Auf die speziellen Schiedskommissionen (Vereins- und Verbandsgerichtsbarkeit), die Schiedsstellen für Arbeitnehmererfindungen, Schiedsgerichte (etwa im Völkerrecht, im Handelsrecht, besonders im Gesellschaftsrecht) wird hier nicht eingegangen, weil sie zu weit vom Thema entfernt sind.) 
gen Beratungs-, Streitschlichtungs- ${ }^{62}$ und Notarfunktionen aus. 34000 von der Bevölkerung "auf den Hügeln" und in den Städten gewählte, also nicht von der Regierung eingesetzte, Notabeln tragen als Bashingantahe auch zur Aufrechterhaltung der öffentlichen Ordnung bei. Ferner nehmen sie Verdächtige vorläufig, d.h. bis zum Eintreffen der Polizei, fest. Dagegen dürfen sie keine Strafen verhängen. ${ }^{63}$

Wegen der hohen Akzeptanz der Einrichtung und der Spruchpraxis der Bashingantahe vor allem bei der ländlichen Bevölkerung "auf den Hügeln" will die burundische Regierung diese Schlichtungseinrichtungen landesweit fördern bzw. wiederherstellen / rehabilitieren / revitalisieren und wird dabei vor allem von der Europäischen Union (EU) und dem Entwicklungsprogramm der Vereinten Nationen (UNDP / PNUD) unterstützt.

Feldbeobachtungen im Dorf Kivyeyi (Gemeinde Kiganda, Provinz Muramvya) ${ }^{64}$ und sogar in der Hauptstadt Bujumbura ${ }^{65}$ mit funktionierendem staatlichem Justizsystem ergaben eine breite Zustimmung zu der Einrichtung.

Es fragt sich allerdings, ob und ggf. wie die Bashingantahe in der derzeitigen kritischen Situation mit der tutsibeherrschten Nationalarmee, einigen noch mit Waffen kämpfenden

Streitschlichtung versucht parallel auf lokaler aber auch auf höheren Ebenen der in der ÜVerf vorgesehene Ombudsman, der aber kaum Breitenwirkung hat. In Deutschland existiert eine solche Einrichtung auf Bundesebene für den Militärbereich: der Wehrbeauftragte des Bundestages (Art. 45 b GG), dessen Erfolge aber z.T. darauf beruhen, dass er in einem sektoralen, überschaubaren Bereich tätig wird.

Das unterscheidet sie von den vergleichbaren Gacaca, "Graswurzelgerichten" im Nachbarland Ruanda, die auch Strafen verhängen dürfen. Seit 2002 wirken sie sogar neben dem Internationalen Kriegsverbrechertribunal für Ruanda (ITCR) in Aruscha (Tansania) und den ruandischen staatlichen Gerichten bei der Ahndung des Völkermords von 1994, bei dem mehr als 800000 Menschen - überwiegend Tutsi - von fanatisierten Hutu ermordet wurden, mit. Die Beteiligung der Gacaca an der Sühne für den Völkermord ist vermutlich dadurch zu erklären, dass die staatlichen ruandischen Gerichte u.a. deshalb besonders langsam arbeiten, weil nahezu alle Richter und Staatsanwälte bei dem Genocid ermordet worden waren. So sollen die traditionellen Gerichte für Beschleunigung der Strafverfolgung von wesentlichen Teilen der 110000 als Tatverdächtige Inhaftierten sorgen; diese müssen sich in ihrer Heimatgemeinde verantworten. Führer und Hintermänner des Völkermords, die sich unter den Untersuchungshäftlingen befinden, sollen allerdings von staatlichen Schwurgerichten abgeurteilt werden. Im Oktober 2001 wurden von den Dorfbewohnern 260000 ehrenamtliche (Laien-) Richter für 11000 Gacacatribunale gewählt. Sprachlich bezeichnet Gacaca auf Kinyaruanda eine Lichtung oder eine lichte Grasfläche, auf der sich die Ältesten treffen, um Streitigkeiten innerhalb der Dorfgemeinschaft zu schlichten. Der versöhnende Charakter der Tätigkeit der Gacaca soll nach der Vorstellung des ruandischen Justizministeriums auch für diese Strafprozesse genutzt werden (vgl. Frankfurter Allgemeine (FAZ) vom 10. Oktober 2001, S. 8).

Vgl. Ndayishinguje, Pascal, The functioning of the bashingantahe institution in a rural village, in: Ntahombaye (u.a.) op. cit. FN 44, S. 107-113. 
oppositionellen Hutugruppen, einer oft betrogenen und daher misstrauischen, unstabilen, ethnisch gespaltenen Bevölkerung, zerstrittenen politischen Parteien, Parteiungen und (Nichtregierungs-) Organisationen, einer im Aufbau befindlichen noch eher hilflosen Zivilgesellschaft, vielen - teilweise zurückkehrenden - Flüchtlingen und einer sich seit 1993 verschärfenden Wirtschaftskrise mit hoher Arbeitslosigkeit wirklich an ihr großes historisches Erbe von/zu adäquaten Problemlösungen anknüpfen (können). Konkret: Können sie zur Abkehr von der Gewalt ${ }^{66}$, d.h. zur Befriedung, zur Versöhnung der Ethnien von Hutu und Tutsi, zur harmonischen, nachhaltigen Entwicklung der Gesellschaft, zur Demokratisierung und zur Verrechtsstaatlichung des zerrissenen Landes auf der lokalen Ebene beitragen?

\section{Kritische Betrachtung}

Rutakes Untersuchung an einem allerdings zahlenmäßig und lokal beschränkten Kreis (202 Personen im Alter von 30 bis 90 Jahren in 82 (von 114) Kommunen) ${ }^{67}$ ergab weit verbreitete Kenntnisse in der Bevölkerung über die Einrichtung der Bashingantahe und deren Bejahung durch die große Mehrzahl der Befragten. Dennoch ist die Einrichtung nicht unumstritten.

Das gilt für einen Teil der Burunderinnen, vor allem für weibliche Intellektuelle. Traditionelle Auffassung ist, dass die Frau ihren Platz im Haus, der Mann seinen Platz draußen hat; danach "kann eine Frau nicht als Richter urteilen" (nta ntahe y'umugore). Dieses traditionelle Rollenverständnis hat sich überholt und Frauen nehmen in der staatlichen Justiz Burundis hohe Positionen, etwa als Präsidentin des Verfassungsgerichts, ein. Und Crescence Ndayiswimiye, Richterin an der Cour constitutionnelle, wies den Verfasser ausdrücklich auf die Ungereimtheit, dass Frauen bei staatlichen Gerichten auf allen Ebenen als Richterinnen tätig, aber für die Bashingantahe nicht wählbar sind, und die damit verbundene Frauendiskriminierung (verboten durch Art. 23 ÜVerf) hin. ${ }^{68}$ Die Präsidentin des Juristinnenbundes (Association des femmes juristes -AFJ), Christine Ntagwirumogaga, und zwei Vorstandsmitglieder der AFJ, Patricia Ntahorubuze und Spès Baransata forderten unter Verweis auf den Gleichheitssatz in der ÜVerf ebenfalls eine Korrektur der Charte des Bashingantahe zugunsten der Aufnahme weiblicher Richter. ${ }^{69}$ Die Gegenseite argumentiert historisch: der (Richter-) Stock als wesentliches Autoritätssymbol des Mushingantahe stelle

66

67

68

69

Vorschläge zur Lösung des Gewaltproblems in der burundischen Gesellschaft: Sindayigaya, J.M., Sortir de la violence au Burundi, Bujumbura 1991, passim, besonders S. $185 \mathrm{ff}$.

Power control and regulation by the bashingantahe institution, in: Ntahombaye (u.a.) op. cit. FN 44, S. 73-87.

Interview am 20. August 2002.

Interview mit dem Verfasser am 20. August 2002. 
ein männliches Symbol dar. Der Nationalrat der Bashingantahe suchte einen Kompromiss: Verheiratete Frauen (bapfasoni) können mit ihren Ehemännern in den Spruchkörper der Bashingantahe aufgenommen werden, ja sogar Witwen und Junggesellinnen können auf Antrag akzeptiert werden, wenn ihr Haushalt aufgenommen wurde. Sie können dann Beraterinnen/Beisitzerinnen (abashigikirantahe) werden. Allerdings sind ihnen Verleihung und Gebrauch des (Richter-) Stocks (intahe) "aus Gründen der Tradition versagt" "70. Diese Position ist den Kritikerinnen zu klein. Und ein anderes historisches Argument, nach dem ein König den Frauen ihr ursprünglich bestehendes (Schieds-) Richteramt (neben männlichen Bashingantahe) weggenommen habe, weil sie das Beratungsgeheimnis nicht eingehalten hätten, ${ }^{71}$ wird von ihnen verständlicherweise nicht akzeptiert.

Nach einer Untersuchung von Barbara Ndimurukundo-Kururu ${ }^{72}$ (allerdings nur) an 127 Befragten (darunter 52 Frauen) forderten 94,80\% von ihnen, Frauen als Vollmitglieder der Bashingantahe zuzulassen.

Man kann sich fragen, ob die Institution der Bashingantahe in Hutukreisen nicht als zu tutsilastig angesehen wird/werden könnte. Sie wurde von einem Tutsikönig eingeführt, diente letztlich der gesellschaftlichen Entwicklung, Mediation und Befriedung im Tutsikönigreich (bis hin zur persönlichen Beratung des Königs) und wurde nach der Unabhängigkeit des Landes von den verschiedenen Republiken eher misstrauisch behandelt. Diesen nicht von der Hand zu weisenden Argumenten ist allerdings entgegenzuhalten, dass es von Anfang an auch Hutubashingantahe gab, dass also zumindest die Idee dieser Schiedsgerichte ethnieübergrei-fend war. Die Zugehörigkeit zur Gruppe der Hutu (und der Tutsi) war zudem nicht allein durch die ethnische Herkunft bestimmt; der Begriff Hutu hatte in der Kirundisprache vielmehr auch eine kulturelle und gesellschaftliche Dimension. So war z.B. am Königshof ein Prinz trotz seiner kulturellen Tutsiidentität in Beziehung zum Mwami ein Hutu. ${ }^{73}$ Auch muss darauf hingewiesen werden, dass es "die" Hutu und "die Tutsi" nicht gab und nicht gibt. Vielmehr setzte sich jede der beiden Ethnien aus verschiedenen Clans zusammen, die überdies in strenger sozialer Hierarchie lebten. Das wird (auch) jetzt besonders deutlich, weil es der (Übergangs-) Regierung trotz mehrerer Versuche nicht gelingt,

1. Abschnitt der Charta der Bashingantahe.

71 Gahama, Joseph, Origin and evolution of the bashingantahe institution, in: Ntahombaye (u.a.) op. cit. FN 44, S. 23 ff. (24). Diesen Standpunkt vertreten auch einige von Barbara NdimurukundoKururu, The Burundian woman and the bashingantahe institution, in: Ntahombaye (u.a.) op. cit. FN 44, S. 137-140 (139) Befragte.

72 The Burundian woman and the bashingantahe institution, in: Ntahombaye (u.a.) op. cit. FN 44, S. 137-140.

73

Klingebiel, Stephan, Burundi (vgl. FN 2), S. 56 unter Bezugnahme auf Lemarchand, R., Burundi: Ethnocide as Discourse and Practice, London 1992. 
die verschiedenen großen und kleinen Gruppen von bewaffneten Huturebellen an einen Verhandlungstisch zu bekommen. ${ }^{74}$

\section{Zusammenfassung und Ausblick}

Bashingantahe sind in BU historisch gewachsene Schiedsgerichte / Friedensgerichte. Sie standen immer für ein Klima der Mediation, nicht der Gewalt. Sie verbanden rechtliche und ethische mit sozialen, alltäglichen, politischen, sogar philosophischen Argumenten und erläuterten sie den Rechtssuchenden in einer für diese verständlichen Sprache. Von ihrer Zusammensetzung und ihrer Aufgabenstellung her können sie auch heute zu effektiver Versöhnung in der burundischen Gesellschaft, etwa bei der (Wieder-) Eingliederung zurückkehrender Flüchtlinge, beitragen. Sie haben nie einem ethnozentristischen Totalitarismus gedient; so stehen sie Tutsi und Hutu offen. Ohne sklavisch der Vergangenheit verhaftet zu sein, können die Bashingantahe "neuer Darstellungsweise" (Bashingantahe "nouveau style") aus reicher Tradition schöpfen. Sie arbeiten basisnah auf der Grundlage von Gewohnheitsrecht, können aber auch geschriebenes und/oder religiöses Recht in ihre Beurteilungen einfließen lassen oder im Hinblick auf ihr Hauptziel der Versöhnung und Befriedung der (Schiedsverfahrens-) Parteien rechtsschöpferisch tätig werden. Sie arbeiten mit hoher Transparenz, d.h. unter Teilnahme der interessierten lokalen Öffentlichkeit, und geringen Kosten in einem einfachen, verständlichen Verfahren. Auch entlasten sie die überlastete, mangelhaft ausgestattete, staatliche Justiz vor allem in den Bereichen des Familien-, Erb- und Bodenrechts sowie des zivilrechtlichen Ehrschutzes. Falls der Streit durch die Bashingantahe nicht geschlichtet / geregelt werden kann, bzw. von mindestens einem der Beteiligten nicht akzeptiert wird, bleibt die Anrufung der staatlichen Justiz offen.

In der besonderen Lage von BU können die Bashingantahe auch in bürgerkriegsberührten (nicht befriedeten) Gebieten Recht sprechen, also in Gebieten, in denen die staatliche Justiz teilweise zum Erliegen gekommen ist. Ein gewisser Pluralismus zwischen Bashingantahe und - vor allem - den Amtsgerichten (Tribunaux de résidence) als unterster Ebene der staatlichen Gerichtsbarkeit (gemessen an der Nähe zu den Rechtssuchenden und den entscheidungsrelevanten Haupt-Rechtsmaterien) ist im Interesse gerechter, von den Rechtsuchenden anerkannter Entscheidungen eher förderlich.

Insgesamt dürfte das burundische Experiment der landesweiten Rehabilitierung der - gewiss noch verbesserungsfähigen/-bedürftigen Einrichtung der Bashingantahe trotz der

Am 14. Juli 2003 haben z.B. Huturebellen von den bewaldeten Hügeln um Bujumbura sieben Raketen auf Wohngebiete in der Hauptstadt abgefeuert und zwei Häuser zerstört. Menschen wurden bei dem Raketenangriff nicht verletzt. Allerdings hat der seit 1993 laufende, nicht erklärte Bürgerkrieg bisher mindestens 200000 Menschen das Leben gekostet (vgl. FAZ v. 16.7.2003, S. 5). 
veränderten Umstände gegenüber ihrer historischen Arbeit und trotz der unterbrochenen Tradition eine reale Chance eines substantiellen Beitrags zur Verbesserung der gesellschaftlichen und staatlichen Entwicklung, der Befriedung, der Versöhnung, der Etablierung des Rechtsstaats und der Demokratie haben. Stellen sich dabei Erfolge ein, kann es sogar als Vorbild für die Anrainerstaaten an den Großen Seen oder in anderen afrikanischen Staaten wirken. 